\title{
Three-Dimensional Computed Tomography From Interferometric Measurements Within a Narrow Cone of Views
}

Arthur J. Decker

Lewis Research Center

Cleveland, Ohio

and

Steven H. Izen

Case Western Reserve University

Cleveland, Ohio

March 1991 


\title{
THREE-DIMENSIONAL COMPUTED TOMOGRAPHY FROM INTERFEROMETRIC MEASUREMENTS WITHIN A NARROW CONE OF VIEWS
}

\author{
Arthur J. Decker \\ National Aeronautics and Space Administration \\ Lewis Research Center \\ Cleveland, Ohio 44135 \\ and \\ Steven H. Izen ${ }^{*}$ \\ Case Western Reserve University \\ Cleveland, Ohio 44106
}

\section{Summary}

A theory to determine the properties of a fluid from measurements of its projections was developed and tested. Viewing cones as small as $10^{\circ}$ were evaluated, with the only assumption being that the property was space limited. The results of applying the theory to numerical and actual interferograms of a spherical discontinuity of refractive index are presented. This theory was developed to test the practicality and limits of using three-dimensional computed tomography in internal fluid dynamics.

\section{Introduction}

Nonintrusive optical measurements and visualization techniques have become increasingly available and important for experiments in internal fluid dynamics (the fluid dynamics of flows in engines), but the viewing ranges are small. Except for special cases such as the flow around helicopter blades (ref. 1), the view typically is restricted to $10^{\circ}$ or less from some principal direction. It is then difficult to measure the spatial distributions of properties such as density and velocity. Current approaches use local measurement methods, such as laser velocimetry (ref. 2) and nonlinear spectroscopy (ref. 3), or two-dimensional measurements based on sheet illumination

${ }^{*}$ NASA-ASEE Summer Faculty Fellow at Lewis Research Center. Part of this work was funded under NASA Grant NAG3-832. (ref. 4). These methods are applied arduously and repetitively to estimate the properties of steady or cyclic flows.

Projective measurement methods such as interferometry, moiré, schlieren, and absorption-emission spectroscopy are well-developed alternatives. Diffuse-illumination holographic interferometry, in particular, is used for multiview recordings of unsteady as well as steady flows. The projective methods are excellent for rapid, whole field, flow visualization (refs. 5 to 8 ), but they are not generally suitable for quantitative analysis of three-dimensional flows unless the projections can be inverted.

There are at least three reasons for making a special effort to solve limited-view inverse problems in internal fluid dynamics. First, it is becoming easy to analyze the essential data: commercial sources of computerized fringe and pattern analysis systems are proliferating. Second, it is becoming easier to record the essential data: lasers such as the $\mathrm{Nd}$ :YAG laser used for holography (ref. 9) and spectroscopy have approached turnkey status. Third, the increasingly sophisticated modeling in internal fluid dynamics demands quantitative data: the distributions of velocity and at least one thermodynamics variable such as density or temperature are required (ref. 10).

There is no reason in principle why a reasonably general function cannot be recovered from its projections in any nonzero viewing range. This conclusion follows from the projection-slice theorem (ref. 11) and the analytic continuation of the partially recovered Fourier transform of the function. However, recovering a function from measurements of a finite number of projections is quite different when the measurements 
are corrupted by noise and systematic effects such as refraction. The problem is extremely ill-posed.

There are in fact two questions being investigated in limited viewing range computed tomography. The first, more fundamental question, asks how well a function with very general properties can be recovered from a finite number of errorand noise-corrupted measurements. The second question asks how much the recovery can be improved by including, for example, constraints such as the values of the function on a surface or a knowledge of the functional forms of the important flow features.

This paper addresses the first question only. A space-limited function with very general properties was assumed. The x-ray transforms, or projections, of that function were assumed to be sampled a finite number of times for a finite number of views. Izen developed the mathematical theory using tomography for this study (refs. 12 to 15). His theory, which expands the function in orthogonal polynomials and employs the projection-slice theorem, uses singular-value decomposition to quantify and combat the ill-posedness (sensitivity to initial value errors) of the inversion procedure. The error in determining the polynomial coefficients is then estimated from the experimental error, and the ill-posedness is controlled by restricting the set of polynomial coefficients actually calculated from data.

This paper begins by summarizing the inversion procedure, which is based on the more general theory presented by Izen. The inversion procedure was first tested on phantoms (mathematical objects). This paper presents the results for a spherical density change and for simulated data from several viewing ranges. The results from two narrow, but perpendicular, viewing ranges are also presented. (Two perpendicular viewing ranges are possible for some wind tunnel experiments.) Finally, the results of testing the inversion procedure on actual measurements are presented. The measurements were from doubleexposure, diffuse-illumination holograms of an approximately spherical density change. Heterodyne interferometry was used to measure 49 fringe patterns from one $10^{\circ}$ half-angle viewing cone for each inversion performed. The experimental technique is discussed, and the results are presented. The results are encouraging, but refraction effects and misregistration errors are especially influential when the viewing range is small. The inversions required substantial computing resources including the NASA Lewis Research Center CRAY X-MP and the NASA Ames Research Center CRAY-2. The work discussed herein is presently being augmented by research into the use of constraints to improve the recovery and the efficiency of recovery of three-dimensional property distributions from their projections within a limited viewing range.

\section{Mathematical Theory}

The goal of this research is to recover the three-dimensional distribution of a physical quantity, such as density, from information on projections of the quantity. Mathematically, the projections are interpreted as line integrals of the object function: the function of three variables representing the physical quantity to be recovered.

Let $f$ represent the object function. We assume that the support of $f$, that is, the region on which $f$ is nonzero, lies within a unit ball in $R^{3}$ and that $f$ is sufficiently regular so that all the integrals discussed will converge. A more precise statement of the required conditions can be found in references 12 and 13. The experimentally available data are samples of the x-ray transform of $f$, defined by

$$
P f(\omega, x)=\int_{\infty}^{\infty} f(x+t \omega) d t
$$

where $\omega \in S^{2}$. That is, $\omega$ is a direction on the unit sphere $S^{2}$. Here $x$ is a point on the corresponding perpendicular plane $\omega^{\perp}$ through the origin. Note that there is no problem with the infinite limits since $f(x)=0$ for $|x| \geq 1$. The projection along a specific direction $\omega$ is defined by

$$
P_{\omega} f(x)=P f(\omega, x)
$$

Scanning the projection of the hologram at a fixed lens position is just sampling $P_{\omega} f(x)$ in $x$. In the continuous case, when $P_{\omega} f(x)$ is known for all $\omega$ and $x, f$ can be recovered by use of the x-ray transform projection slice theorem (ref. 11). This theorem is the fundamental relation between the $\mathrm{x}$-ray transform and the object function which is used to recover the object function from the holographic data. Before presenting the theorem we need to establish some notation conventions. The $n$-dimensional Fourier transform (here $n$ is either 2 or 3 ) is defined as

$$
F_{n} f(x)=\int_{\mathbb{R}^{n}} e^{-i x \cdot k} f(x) d x
$$

and correspondingly, the inverse Fourier transform is given by

$$
\left(F_{n}^{-1} f\right)(k)=(2 \pi)^{-n} \int_{\mathbb{R}^{n}} e^{i x \cdot k} f(k) d k
$$

\section{THEOREM (Projection-Slice)}

Fix $\omega \in S^{2}$. Then for $\eta \in \omega^{\perp}$,

$$
(2 \pi)\left(F_{3}^{-1} f\right)(\eta)=\left(F_{2}^{-1}\left(P_{\omega} f\right)\right)(\eta)
$$

For a proof, see references 12,16 , or 17 . Here the twodimensional inverse Fourier transform of $P_{\omega} f$ is performed on the plane $\omega^{\perp}$. The result is also a function on $\omega^{\perp}$. The projection-slice theorem states that this function on $\omega^{\perp}$ is really just the inverse three-dimensional Fourier transform of the object function evaluated on a slice, $\omega^{\perp}$, of $R^{3}$. Thus 
each direction $\omega$ on which projection data is acquired gives rise to data for the inverse Fourier transform of $f$ on the corresponding orthogonal plane through the origin. At this point we would like to point out that the inverse Fourier transform was used instead of the Fourier transform in order to simplify tracking signs. Except for the inconsequential sign of the argument, the two transforms are equivalent, so there should be no confusion when we refer to the inverse Fourier space as Fourier space.

Now we describe the algorithm based on a discretization of the above theorem, said theorem applying to continuously sampled data. The algorithm must take into account the discrete nature of the sampled data and must also employ a satisfactory discrete representation for the object function.

The discretization of the object function $f$ is obtained by expanding $f$ in a generalized Fourier series

$$
f(r, \varphi, \theta)=\sum_{s=0}^{\infty} \sum_{m_{0}=0}^{s} \sum_{m_{1}=-m_{0}}^{m_{0}} a_{s}^{m_{0}, m_{1}} V_{s}^{m_{0}, m_{1}}(r, \varphi, \theta)
$$

for

$$
s-m_{0} \text { even }
$$

where $\left\{V_{S}^{m_{0}, m_{1}}\right\}$ is an orthogonal basis for square integrable functions in $R^{3}$ supported in the unit ball, and $\left\{a_{s}^{m_{0} m_{1}}\right\}$ are the unknown coefficients to be recovered. More specifically, $V_{s}^{m_{0}, m_{1}}$, in polar coordinates $(r, \varphi, \theta)$, can be expressed in terms of the spherical harmonics $S\left(m_{0}, m_{1} ; \varphi, \theta\right)$ and the Jacobi polynomials $P_{n}^{(s, t)}$ by

$$
\begin{gathered}
V_{s}^{m_{0}, m_{1}}(r, \varphi, \theta)=R_{s}^{m_{0}}(r) S\left(m_{0}, m_{1} ; \varphi, \theta\right) \\
R_{S}^{m_{0}}(r)=r^{m_{0}}(w(r))^{-1} P_{\left(s-m_{0}\right) / 2}^{\left(m_{0}+1 / 2-3 / 2\right)}\left(1-2 r^{2}\right)
\end{gathered}
$$

and

$S\left(m_{0}, m_{1} ; \varphi, \theta\right)=\left(\frac{\left(2 m_{0}+1\right)\left(m_{0}-m_{1}\right) !}{4 \pi\left(m_{0}+m_{1}\right) !}\right)^{1 / 2} P_{m_{0}}^{m_{1}}(\cos \varphi) e^{i m_{1} \theta}$

where $P_{n}^{m}$ is the associated Legendre function, and $\alpha$ is an arbitrary parameter controlling the relative weight given to data from near the origin compared with data from near the boundary $|r|=1$. The factor $w(r)=\left(1-|r|^{2}\right)^{-(\alpha-3 / 2)}$ is required to maintain orthogonality.

The three-dimensional inverse Fourier transform of $V_{s}^{m_{0}, m_{1}}$ is computed analytically in reference 12 . Applying that transform to equation (1) gives

$$
\begin{aligned}
F_{3}^{-1} f(q, \mu, \tau)=\sum_{s=0}^{\infty} \sum_{m_{0}=0}^{s} \sum_{m_{1}=-m_{0}}^{m_{0}} a_{s}^{m_{0}, m_{1}} c_{\alpha, s, m_{0}} \\
\times\left[q^{-\alpha} J_{\alpha+s}(q) S\left(m_{0}, m_{1} ; \varphi, \theta\right)\right]
\end{aligned}
$$

for

$$
s-m_{0} \text { even }
$$

where $(q, \mu, \tau)$ are spherical coordinates on the inverse Fourier $\mathrm{R}^{3}, J_{v}(t)$ is the Bessel function of the first kind, and

$$
c_{\alpha, s m_{0}}=i^{m_{0}}(2 \pi)^{-3 / 2} 2^{\alpha-3 / 2} \frac{\Gamma\left[\left(s-m_{0}\right) / 2+\alpha-1 / 2\right]}{\Gamma\left[\left(s-m_{0}\right) / 2+1\right]}
$$

A finite discretization for $f$ is accomplished by terminating the first sum in equation (2) at $S$. In this case, there are $(S+1)(S+2)(S+3) / 6$ unknown coefficients $a_{s}^{m_{0}, m_{1}}$ to be determined. Once these coefficients have been recovered, then $f$ can be reconstructed from equation (1). Such a reconstruction is said to be of degree $S$ because

$$
\left\{w(r) V_{s}^{m_{0}, m_{1}} \mid s \leq S, s-m_{0} \text { even, }\left|m_{1}\right| \leq m_{0}\right\}
$$

is a basis for the polynomials of degree $\leq S$. In other words, the terminated sum representation of $w(r) f$ is a degree $S$ threedimensional Taylor series.

The data are not available on a continuum, but rather are only sampled discretely on each $\omega^{\perp}$. Substituting a twodimensional, discrete, inverse Fourier transform for the continuous transform on each $\omega^{\perp}$ gives a new sampling grid on $\omega^{\perp}$ for $F_{2}^{-1}\left(P_{\omega} f\right)$ which, by the projection slice theorem, is interpreted as being on a slice in the Fourier $R^{3}$. Thus, if

$$
\left\{\eta_{i} \mid i=1, \ldots, I\right\}
$$

is the set of such sample points in Fourier $R^{3}$, then

$$
F_{3}^{-1} f\left(\eta_{1}\right)=\sum_{s=0}^{\infty} \sum_{m_{0}=0}^{S} \sum_{m_{1}=-m_{0}}^{m_{0}} a_{s}^{m_{0}, m_{1}} F_{3}^{-1} V_{s}^{m_{0}, m_{1}}\left(\eta_{i}\right)
$$

for

$$
s-m_{0} \text { even }
$$

where $F_{3}^{-1} f\left(\eta_{i}\right)$ is computed directly from the experimental data by using a two-dimensional fast Fourier transform, and $F_{3}^{-1} V_{s}^{m_{0}, m_{1}}\left(\eta_{i}\right)$ can be directly evaluated since the location of $\eta_{i}$ is explicitly known, as is the analytic expression for $F_{3}^{-1} V_{s}^{m_{0}, m_{1}}$. 
Thus the inversion procedure has been reduced by a careful discretization to the solving of a linear system

$$
\vec{b}=Q \vec{a}
$$

where $\vec{b}$ is the column vector of $I$ elements $F_{3}^{-1} f\left(\eta_{i}\right), \vec{a}$ is the $(S+1)(S+2)(S+3) / 6$ element vector of unknown coefficients, and $Q$ is the matrix of basis functions $F_{3}^{-1} V_{s}^{m_{0}, m_{1}}$ evaluated at the sample points $\eta_{i}$. Note that $\vec{b}$ depends on the sampling geometry and the measured data, while $Q$ depends only on the sampling geometry and the desired degree of the reconstruction.

In the following discussion, by data we refer not to the raw experimentally obtained data, but rather the data after the twodimensional Fourier transforms have been applied. Practical difficulties arise in numerically solving the linear system (eq. (3)). First, experimental considerations restrict the set of directions from which projection data are available. This means that only a very small portion of three-dimensional Fourier space is covered with sample points. This is manifested as a severe ill-posedness in the linear system. The method employed to combat the ill-posedness and the resulting sensitivity to noise is the application of the singular value decomposition (SVD) to obtain a least squares solution for $\vec{a}$. The ill-posedness means that the solution to equation (3) is much more sensitive to certain components of the data than others. Thus, small amounts of noise in some data components can swamp the solution. The SVD provides a mechanism to eliminate this problem. A decision is made as to what level of relative sensitivity is acceptable given the noise present in the data, and no components of the data space for which the sensitivity exceeds this cutoff are used in the reconstruction. While eliminating unwanted noise sensitivity, it has the drawback of giving an incomplete solution in the sense that the component of the solution corresponding to excised data is missing. However, this component could not be reliably recovered anyway, so the resulting solution is, in some sense, the best that can be done under the circumstances. Many references on the SVD and ill-posed problems can be found in the literature (ref. 17, for example).

The second practical problem in implementing the algorithm is the size of the reconstruction. Each projection was sampled with a relatively low density of 32 by 32 , and data from 49 projections were collected. In practice, only the central 29 of these projections were used, so for this experiment $I=29696$. In order to obtain useful results on experimental data, the order of the reconstruction needs to be at least $S=12$, so there are 455 coefficients to be determined. Thus, the matrix $Q$, on which the SVD must be performed, has 13.5 million entries, each of which requires 16 bytes (double precision complex). Therefore, each reconstruction requires over 200 megabytes of memory, even for modest values of the parameters $I$ and $S$ controlling the reconstruction size. This SVD is computationally expensive to perform, even after vectorization. Fortunately, since the matrix $Q$ only depends on the sample geometry, it can be computed just once and used repeatedly for different data sets.

The computational needs for the inversion were met by the NASA Ames Cray-2 supercomputer. A typical run of the size described takes approximately $5000 \mathrm{sec}$ of single CPU processing, most of which is spent on calculating the SVD of $Q$.

\section{Numerical Experiments}

Numerical experiments were performed to test the implementation of the reconstruction algorithm. Computergenerated data were reconstructed under various conditions, some corresponding to sampling geometries obtainable in the laboratory, and others corresponding to more general circumstances. The results reported in this section are for the numerical reconstruction of a model ball. Projection data were computed for an object which was unity on the interior and zero on the exterior of a sphere centered at the origin with a diameter of one-half the field of view. This model ball appears in figure 1 .

All of the reconstructions are displayed as contour surfaces with a constant function value and as slices through the object, again using color to indicate function value. The rectangular jagged edges at the boundary of the displayed region are an artifact produced by the display software handling the blanked points outside the spherical field of view. These jagged edges have no scientific significance. The figures were created with the software PLOT3D on a Silicon Graphics Personal Iris 4D/25 graphics workstation and a Seiko Instruments CH-5504-PM3 printer, together with the Freedom of Press PostScript interpreter.

In the first reconstruction, projection data from a $90^{\circ}$ cone, that is, a full view, were used. This numerical experiment shows that the algorithm can perform accurately (to within the limits imposed by the sampling density) when data from all directions are available. The reconstruction shown in figures 2 to 4 was done to degree 12 . That is, $S=12$, and 455 coefficients $a_{s}^{m_{0}, m_{1}}$ were determined. This reconstruction is very well-posed since the condition number (the ratio of the largest to smallest singular value) for the matrix $Q$ is approximately 5 . In viewing figures 2 to 4 , note that the spherical symmetry of the original model has been retained.

In the second experiment, a $10^{\circ}$ viewing cone along the $z$-axis was used. In this case, data was generated for the projections from 29 viewing directions within that cone. This configuration corresponds to the sampling geometry of the laboratory experiment discussed later. The condition number for $Q$ with this set of sample directions is $6.72 \times 10^{12}$, which confirms that this is an ill-posed problem, as expected. Figure 5 exhibits a couple of contour surfaces for the resulting image, and figure 6 shows three central cross sections. The spherical model appears here as an oblong object. The cross section along the $x y$-plane, roughly perpendicular to the viewing directions, is circular and faithfully reproduces the cross 


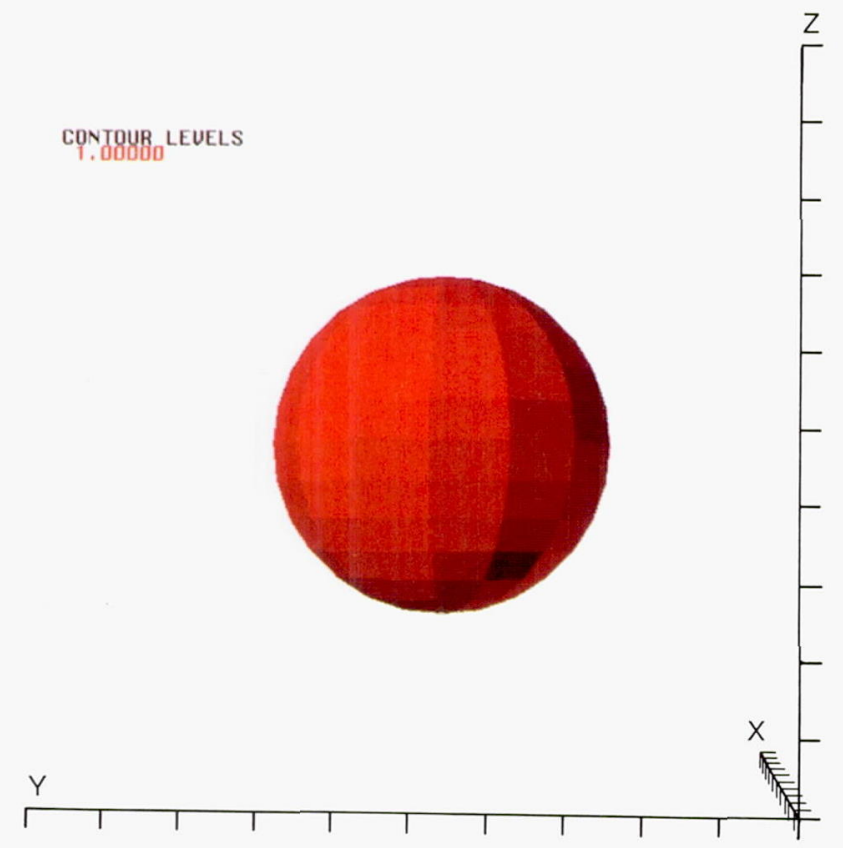

Figure 1.-Model ball from which theoretical data is generated. The ball is located at the center of the field of view and has a diameter of one-half the field of view.

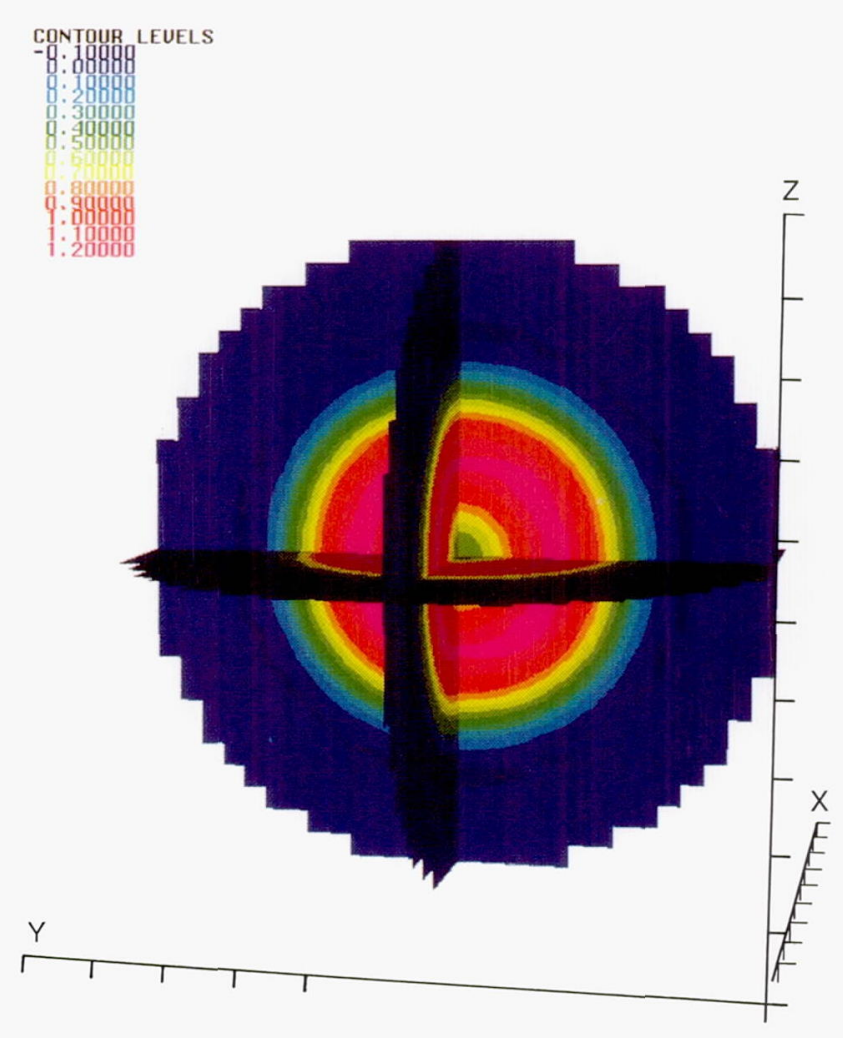

Figure 3.-Three orthogonal slices through the center of the full-view reconstruction. Note that the edge of the model ball is fairly accurately located in all of the planes. There is also some Gibbs phenomenon present.

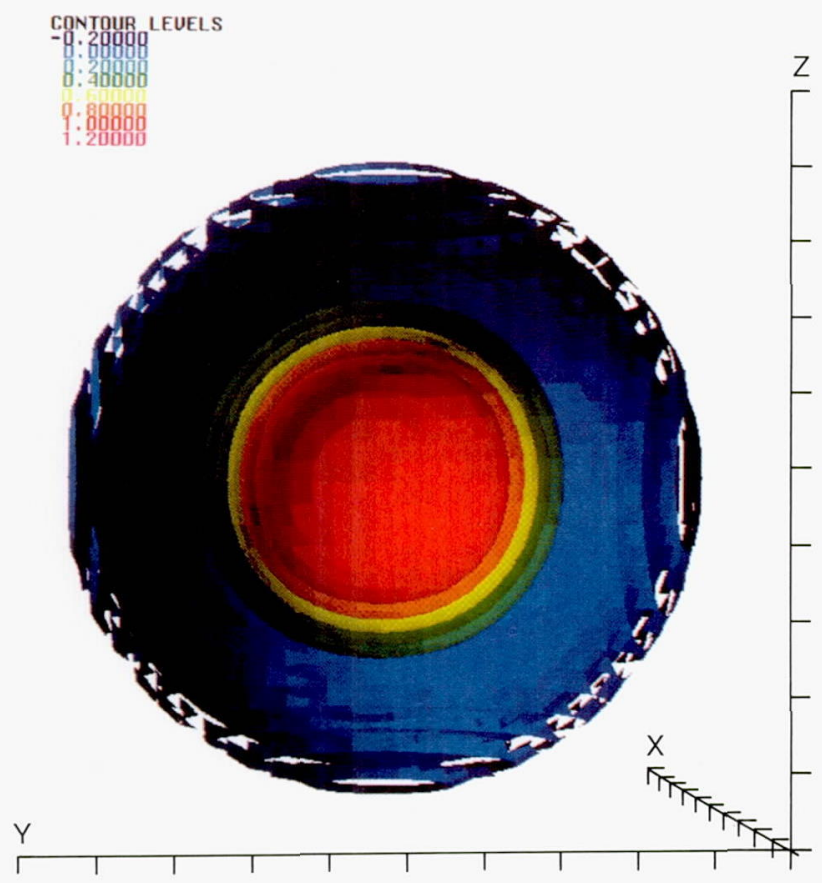

Figure 2.-Full-view reconstruction of the model ball. The views used in the reconstruction were all sampled in a cone of central angle $90^{\circ}$; that is, there were no restrictions on the viewing directions. The jaggedness appearing near the edge of the field of view is an artifact of the contouring and display algorithm, and does not appear in the data.

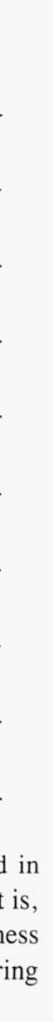

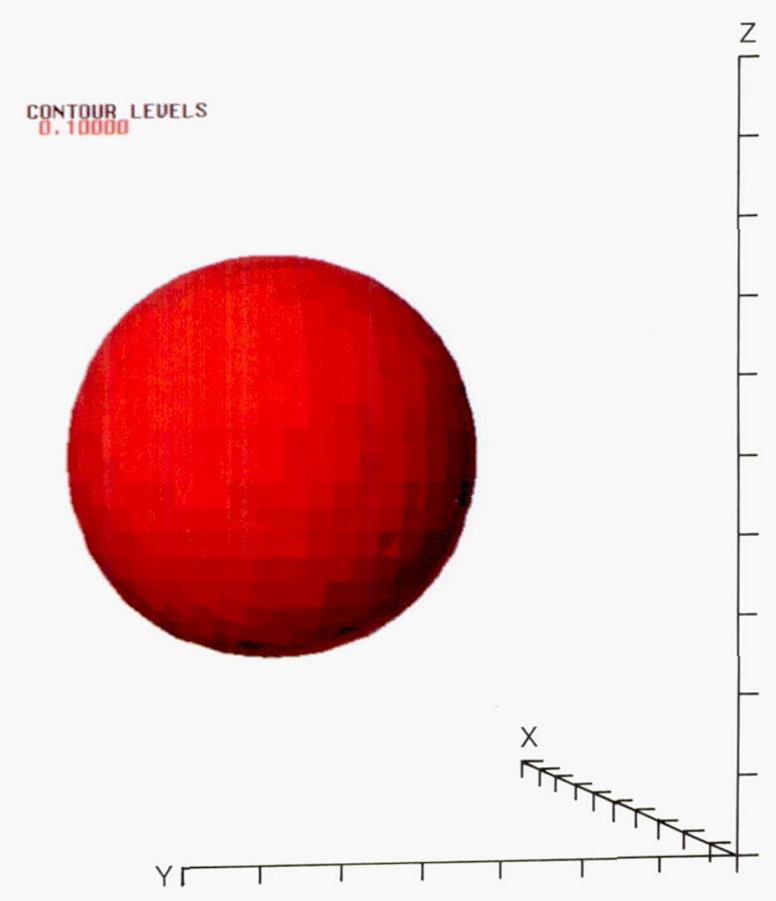

Figure 4.- One contour surface of the full-view reconstruction near the expected boundary of the model ball. The spherical nature of the model ball has been recovered. 


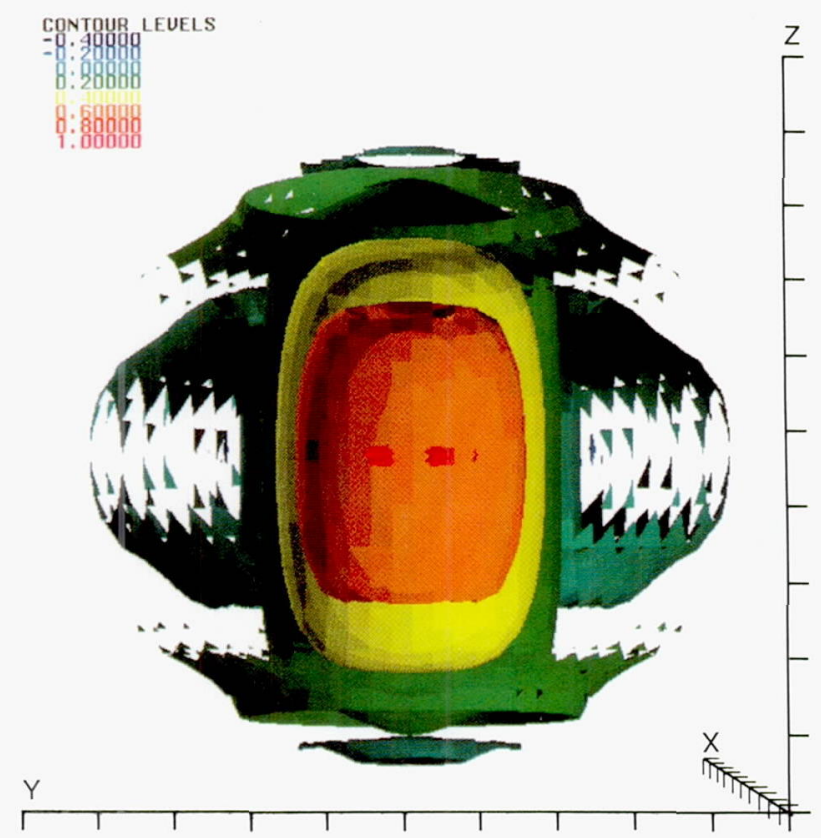

Figure 5.- Reconstruction of the model ball from projections all lying inside a cone of central angle $10^{\circ}$. The axis of the viewing cone or cone of projection directions is along the $z$-axis. The jagged edges are again an artifact of the contour and display algorithm. For a $10^{\circ}$ reconstruction, the method of this paper appears to be unable to recover the boundary locations along directions approximately parallel to the projection directions.

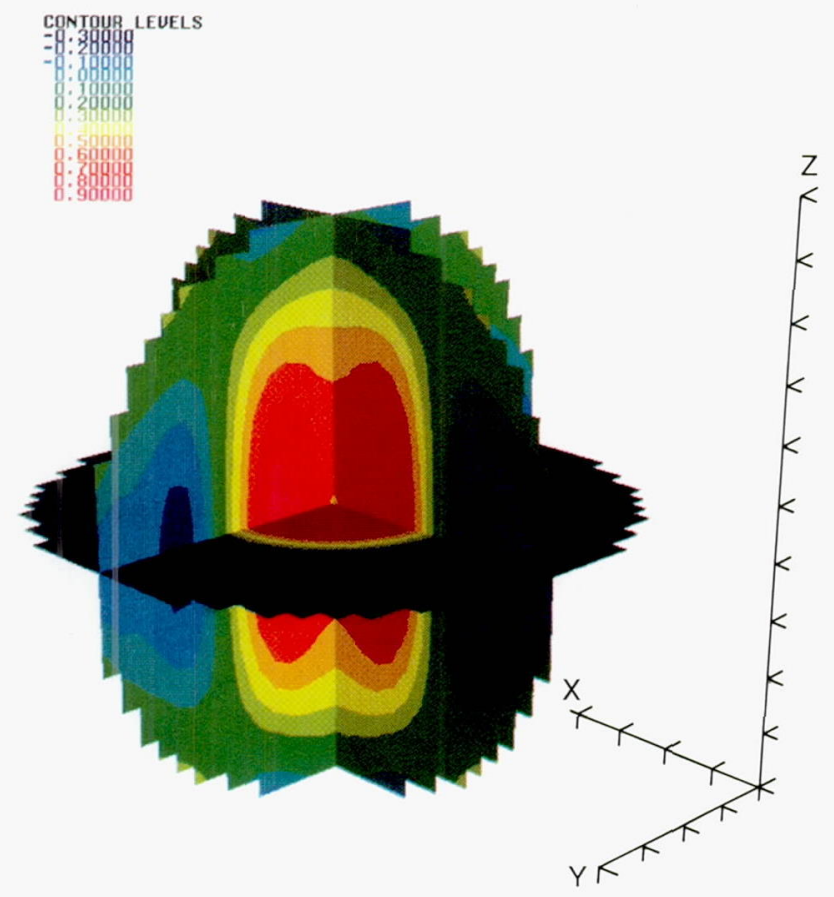

Figure 6.- Three orthogonal slices through the same reconstruction as in figure 5 . Note that along the $x y$-plane, the slice accurately locates the model boundary, but along the $x z$-and $y z$-planes it shows elongation in the $z$-(projection) direction. section of the model (again, to within the limits imposed by the sampling). However, there is an elongation along the $z$-axis, parallel to most of the viewing directions. This loss of resolution, the corresponding smoothing, and uncertainty in the position of the edge location is interpreted geometrically as being due to the lack of data for lines which are tangent to the edge. Analytically, this phenomenon occurs because some of the basis function coefficients for the object space have been zeroed so as not to make the solutions sensitive to noise. In fact only the singular vectors corresponding to 255 out of 455 of the singular values, the ones within a factor of 50 of the largest singular value, were used. The factor of 50 was chosen because the signal-to-noise ratio in the laboratory data was expected to be at worst 50 . The components in the object function space which were omitted are the ones with higher spatial frequency content in the $\mathrm{z}$-direction, precisely the ones which are needed to display sharp boundaries in that direction. In figure 7, one particular contour surface is shown to illustrate the severe distortion that can occur.

One possible experimental setup would permit two separate holographic plates to be exposed simultaneously. If these plates are placed at right angles to one another it would be possible to have data from two orthogonal viewing cones available for the reconstruction. The third numerical experiment was designed to explore whether there would be sufficient improvement to justify the construction of such an experimental apparatus. Here, data from two viewing cones, each with 29 viewing directions, were generated. The reconstruction becomes much less ill-posed, with a condition number of 1431, and with 417 singular values within a factor of 50 of the largest. Thus, the model object can be recovered more accurately. Another interpretation of this result is that a significantly larger region of three-dimensional Fourier space is populated with sample points than is covered in the second experiment. The region of Fourier space which is populated by two cones of sample points can be even more important than the total volume covered. In particular, one $20^{\circ}$ viewing cone is inferior to the two orthogonal $10^{\circ}$ cones, despite the larger volume sampled inside of the $20^{\circ}$ cone. Figures 8 to 10 display the model ball reconstructed with data from two orthogonal $10^{\circ}$ viewing cones. Here, a good portion of the spherical nature of the model has been recovered, but there is still a loss of resolution due to the incomplete coverage of Fourier space. The elongation present in figures 5 to 7 has been significantly reduced because there are now data available from lines tangent to the $x y$-plane. This allows the determination of boundaries which are nearly parallel to that plane. However, some shape artifacts remain as a result of the incomplete Fourier space coverage. In particular, the central slices appear to have developed corners, and in figure 10, the single contour displayed assumes the shape of a cubeoctahedron. As of a result of this numerical experiment, a decision was made to incorporate two windows into a wind tunnel. This will make it possible to obtain two cones of projection data experimentally. 


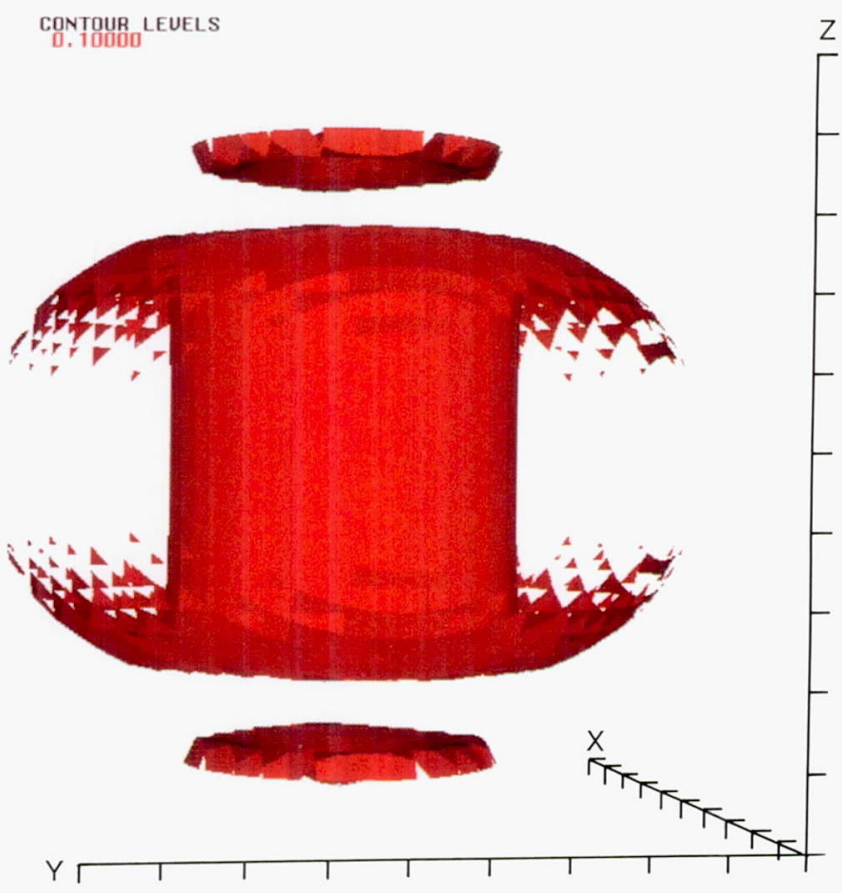

Figure 7.-The same contour level shown in figure 4 for the full-view reconstruction is shown here for the $10^{\circ}$ reconstruction. The caps at the ends appear to be a form of Gibbs phenomenon. The ball appears to "leak" out through the ends.

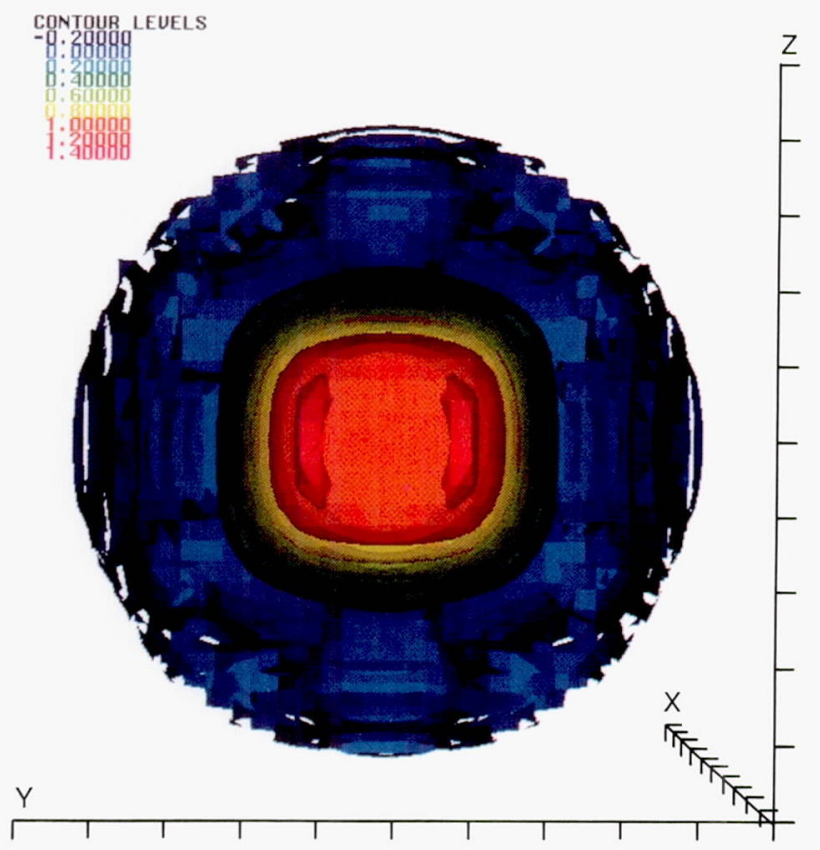

Figure 8.-Projection data from directions inside two orthogonal $10^{\circ}$ cones, one along the $x$-axis, the other along the $z$-axis, were used to produce this image. There is considerable improvement, compared with figure 5, in the recovery of the edge locations.

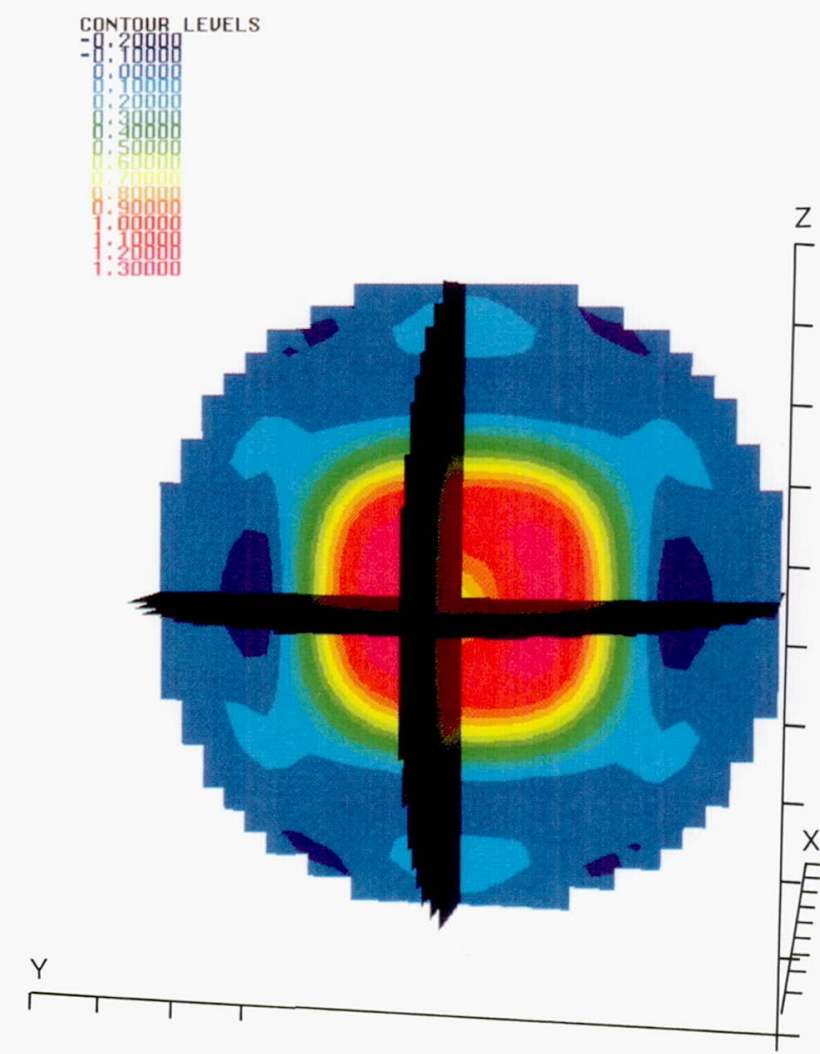

Figure 9.-Three orthogonal slices through the two-cone reconstruction. There is a slight squaring artifact visible, and the contours in the region outside the model ball have become irregular.

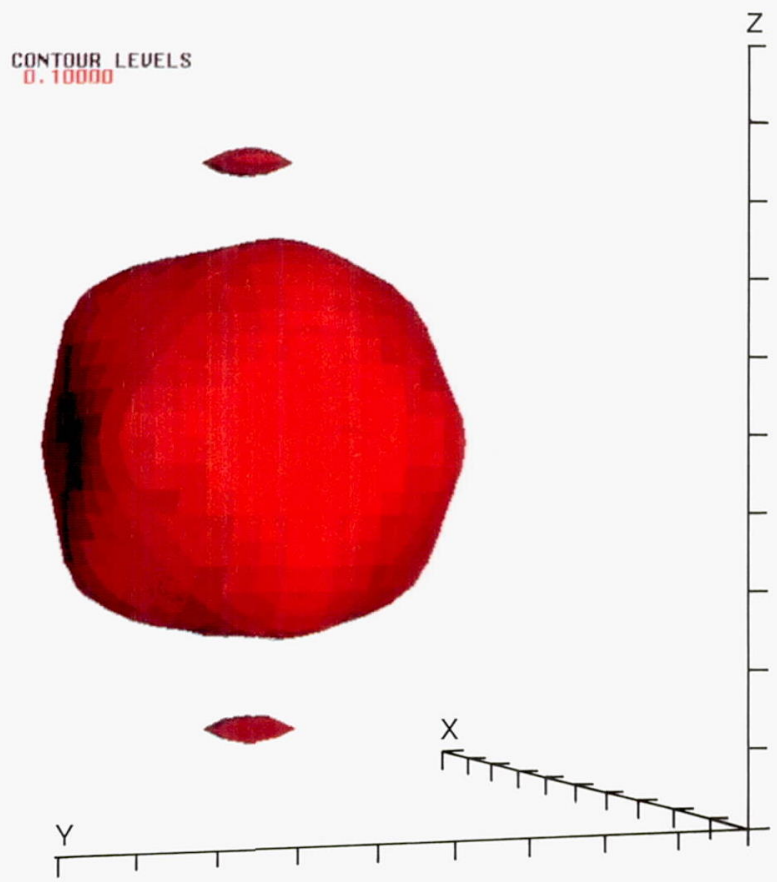

Figure 10.- The same contour shown in figures 4 and 7 is shown for the two-cone reconstruction. The shape of the contour closely resembles a cubeoctahedron, which explains the squaring artifact appearing in the orthogonal slices through the center (fig. 9). The caps at the ends appear to be a form of Gibbs phenomenon. 


\section{Experimental Procedure}

This section discusses an experiment to create and measure a real object that simulates the numerical object, or phantom. For the experiment, we attempted to create a spherically bounded change in index of refraction, said change occurring between the two exposures of a double-exposure hologram. The reconstructed projections or fringe patterns were then measured for 49 views within a limited viewing range, and the measurements were subjected to the inversion procedure for comparison with the phantom data. For consistency with the numerical experiment, only 29 views were used.

The object used was a liquid (dimethyl sulfoxide, or DMSO) contained in a rectangular cell having inside height, width, and length of 106, 136, and $51 \mathrm{~mm}$ respectively. A $50-\mathrm{ml}$ Pyrex spherical flask, having an outside diameter of about $48 \mathrm{~mm}$, divided the liquid into two regions. Two glass windows, $92-\mathrm{mm}$ high by $110-\mathrm{mm}$ wide by 6 -mm thick, allowed diffused laser light to pass through the cell to a 4 - by 5 -in. holographic plate located $241 \mathrm{~mm}$ from the flask center. The flask was centered with respect to the holographic plate, and clamped with its neck vertical. The DMSO $\left(n_{D}=1.4773\right.$ at $25{ }^{\circ} \mathrm{C}$, ref. 18) was index-matched visually to Pyrex by adding a small amount of water.

Double-exposure, diffuse-illumination holograms were used to record changes in the index of refraction of the liquid. The reconstructed image of the flask and the fringe patterns were then imaged (unity magnification) at a detector plane by an $\mathrm{f} / 7.5$ lens. The lens was mounted close to the hologram in an actuator-driven, $x y$-translation stage. The lens could be positioned with micrometer sensitivity within an $82-\mathrm{mm}$ by $82-\mathrm{mm}$ window that was parallel to the hologram. The half angle of the cone of views of the center of the flask was then about $10^{\circ}$.

The fringe patterns were detected and measured by optical heterodyning (ref. 20). Dual reference beam holograms were recorded for this purpose with an argon-ion laser. The reference beam interangle was $0.015^{\circ}$, and the reference signal for the phase meter was derived from the interference pattern (about one-half fringe per millimeter) formed by the reconstruction beams. A lock-in amplifier was used to make the interference-phase measurements. A 1.5-mm-diameter fiber bundle and photomultiplier were combined to detect the fringe pattern. The fiber bundle was positioned with an accuracy of $10 \mu \mathrm{m}$ by a three-axis computer-controlled positioning stage. The same computer was used to collect and process the measurements. This setup, with the small angle between reference beams, has a sensitivity of about 1/100 fringe.

The maximum phase variation between the center and edge of the image of the flask was restricted to about one fringe $\left(360^{\circ}\right)$. Even one fringe corresponds to a fringe gradient, averaged over the detector, of $85^{\circ} / \mathrm{mm}$ at the edge.

It is difficult to produce a uniform change that yields one fringe. The index of refraction of the liquid in the flask depends on composition, temperature, and pressure (refs. 18 and 19).
Solvents, except for water, have large temperature coefficients of index of refraction. The temperature effect for the flask of DMSO is about 20 fringes $/ \mathrm{K}$. For this experiment, a pump was used to halve the pressure in the flask between exposures of the double-exposure hologram. The pressure reduction acts to decrease the index of refraction, but the accompanying temperature decrease acts to increase the index. The net effect was, fortuitously, a center-to-edge phase change of about a fringe.

Measurements were made at 49 views corresponding to 49 uniformly spaced lens positions within the $82-\mathrm{mm}$ by $82-\mathrm{mm}$ window of lens positions. An earlier experiment done with water had shown the importance of having good registration of the different views. To assure registration, a point light source was centered in the reconstructed image of the flask, and the starting point of the detector was always set relative to the image of that light source. The uncertainty of this procedure was estimated to be $0.2 \mathrm{~mm}$. Measurements were recorded for each view at 32 by 32 uniformly spaced points within a $55-\mathrm{mm}$ by $55-\mathrm{mm}$ region. This region included part of the neck of the flask as well as some points in the surrounding fluid. Figure 11 is a plot of the data for a sample view. All data include a phase offset that depends on the measurement electronics and the reference fiber position. A phase measurement at a point in the surrounding fluid must be used as a reference.

There are a number of effects and errors that might affect the results: the random and systematic phase-measurement errors of heterodyne holographic interferometry (ref. 20) averaging over the detector aperture; variations in spherical perspective within a view; the effects of index mismatch including reflection, refraction, and interexposure motion of the flask; nonuniformity of the change in refractive index;

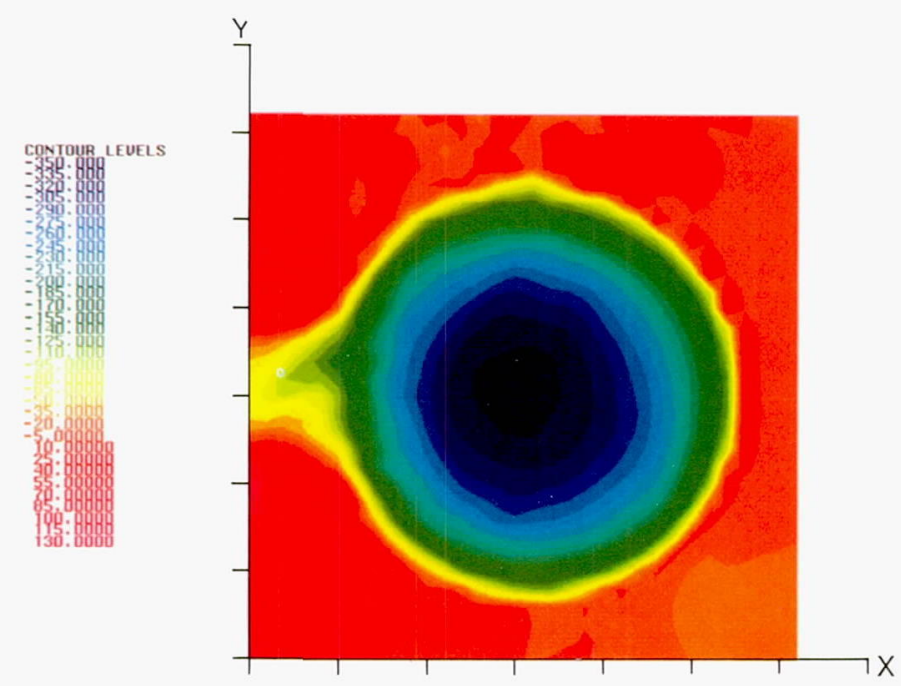

Figure 11.-Experimental data from the hologram. The central projection (along the axis of the viewing cone) is displayed. The data from this and 28 other projections were used to generate the following three figures. 
distortion; and misregistration of the views. An earlier experiment performed with water as the working fluid showed that refraction and misregistration have the most serious consequences.

A set of measurements was performed on the DMSO hologram to show the effect of misregistration. A small viewto-view variation was superimposed on the starting position of the detector. The variation, equal to the spacing between measurements $(1.774 \mathrm{~mm})$, was applied randomly in the horizontal or vertical scan directions.

The results for the registered and misregistered data are presented in the next section and are compared with the results for the equivalent phantoms.

\section{Experimental Results}

In this section we describe the reconstruction obtained from the experimentally acquired data and contrast the results with

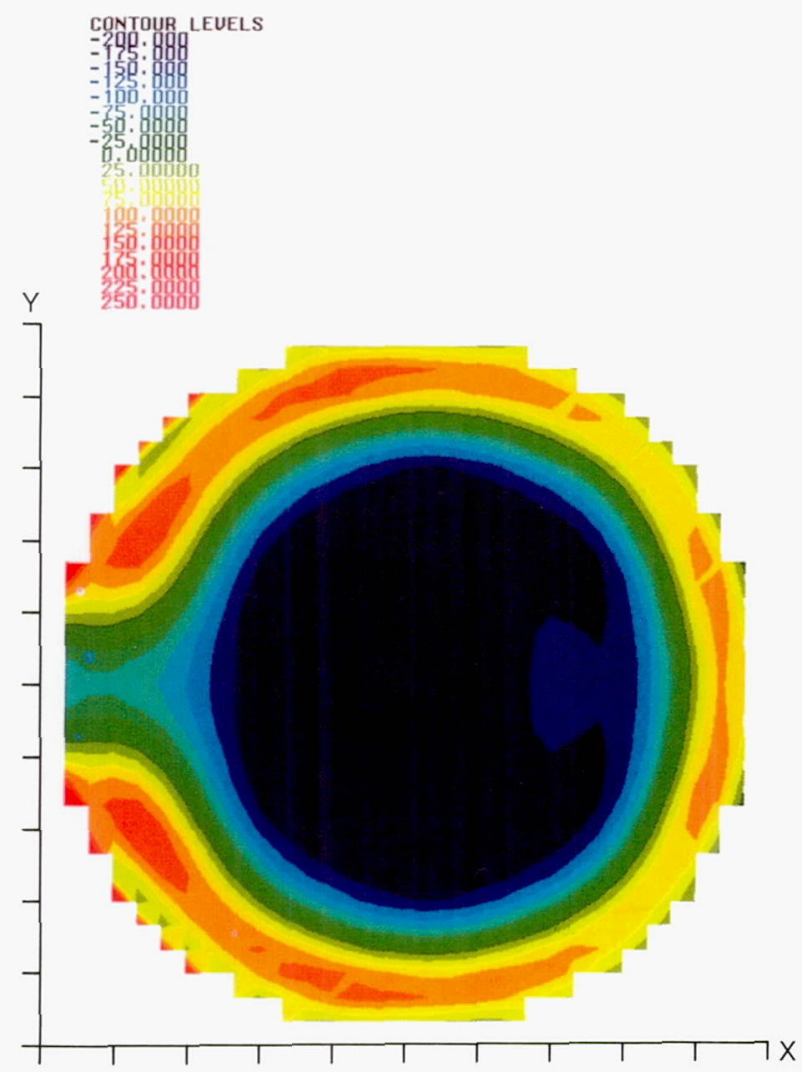

Figure 12.-Slice along the $x y$-plane through the center of the field of view of the reconstruction of a small spherical flask with a short neck. The data were sampled in a $10^{\circ}$ viewing cone by the methods described in the text. The neck of the flask appears in the reconstruction at the lef of the image. This is approximately the view obtained by looking directly at the hologram. the numerical experiments performed on computer-generated data.

Using the central 29 views of the experimental data, a degree 12 reconstruction was performed. The central projection of the experimental data is shown in figure 11. The profile of the flask is visible. Also note that the body of the flask is only approximately spherical, because of either experimental error or aberrations in the flask itself.

Since the sampling geometry and reconstruction degree were the same as in the second numerical experiment described in section 3 (figs. 5 to 7), the SVD did not need to be recomputed. Thus, the reconstruction of the experimental data required only 200 additional sec of CRAY-2 CPU time. Figures 12 to 14 show the results. In figure 12 , we see a slice through the center of the field of view on the $x y$-plane. This plane is parallel to the plane of the hologram, and the flask is recovered faithfully. The green-yellow contour, of value zero, corresponds to the physical edge of the flask. The location and size of the body and neck of the flask are well reproduced, although there is Gibbs phenomenon (ringing) and some smoothing present in

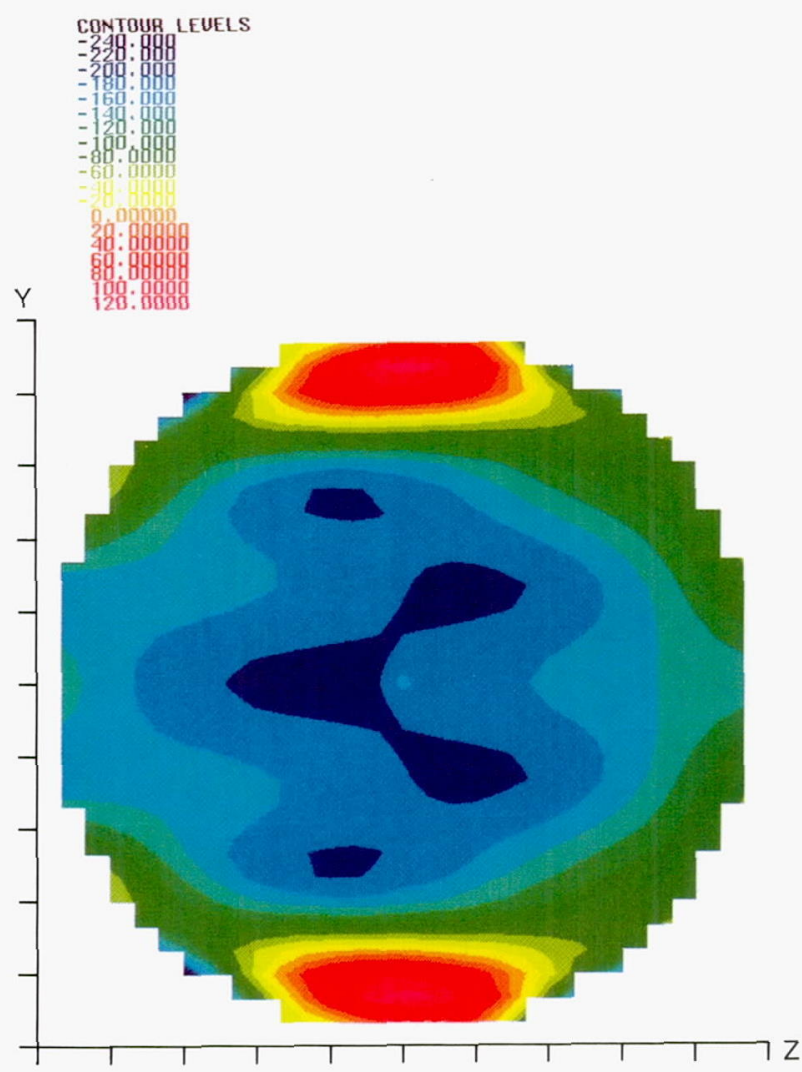

Figure 13.--Slice through the flask reconstruction along the $y z$-plane. This view corresponds to looking down the neck of the flask. Along the $y$-direction the edges are well defined, but in the $z$-direction, the projection direction, the edges have been smeared out. 


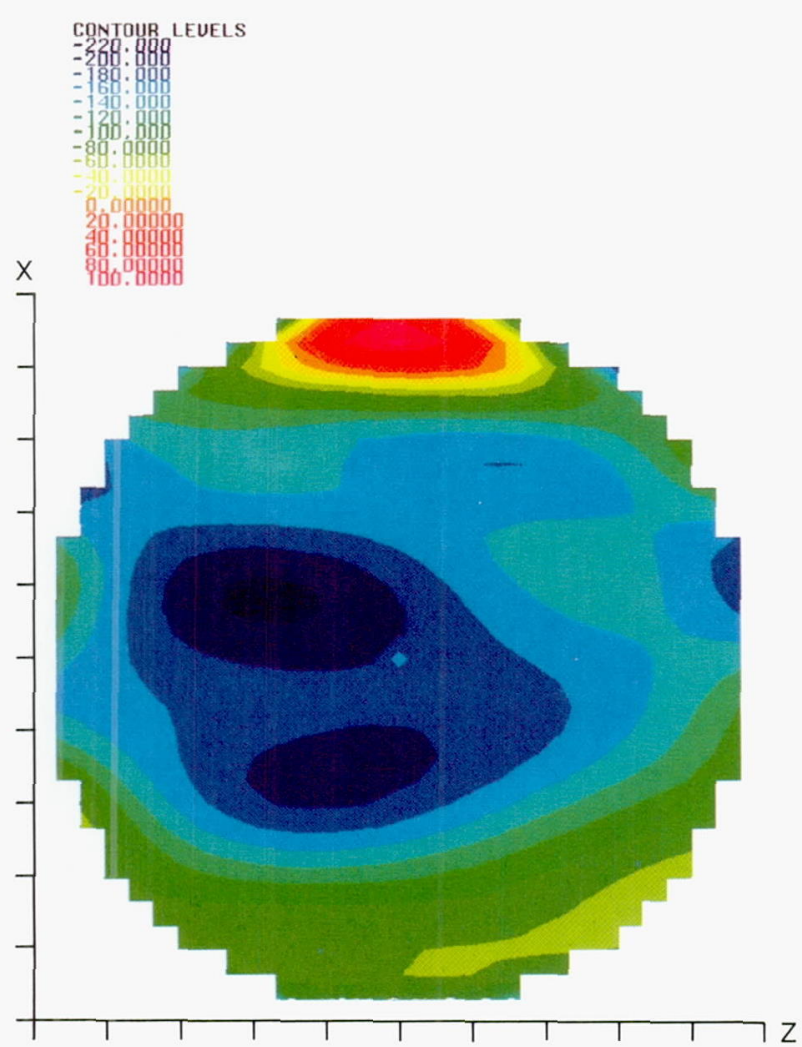

Figure 14.- $x z$-slice of the flask reconstruction. The edge at the bottom of the flask, opposite the neck, is recovered. However, the neck edges are not well recovered. The neck appears as the spread-out green region on the bottom of the image. The body of the flask consists mainly of the cyan region.

the reconstruction. In figures 12 and 13 , sections of the reconstructed image are shown on the planes transverse to the hologram. Along these planes, significant artifacting is present, and the edge locations suffer from blurring. In the experimental data it is assumed that the noise level is at most 2 percent. The SVD is used (keeping all singular values within a ratio of 50 of the largest) to combat the ill-posedness of the reconstruction in the presence of noise. The effect of this procedure appears as a reduction in the rate of increase of function value when crossing the boundary of the flask from the interior to the exterior. In fact the rate becomes so slow in the directions transverse to the $x y$-plane that the function value external to the flask is not achieved before the end of the field of view is reached. The same phenomenon is observed along the $z$-axis in the $10^{\circ}$ viewing cone numerical phantom reconstruction (figs. 5 to 7). It is anticipated that constraint data such as that which could be obtained from measurements using sheet illumination could greatly improve the reconstruction quality. If such additional data provide linear constraints, they can easily be incorporated into the reconstruction algorithm as additional rows in the matrix $Q$.

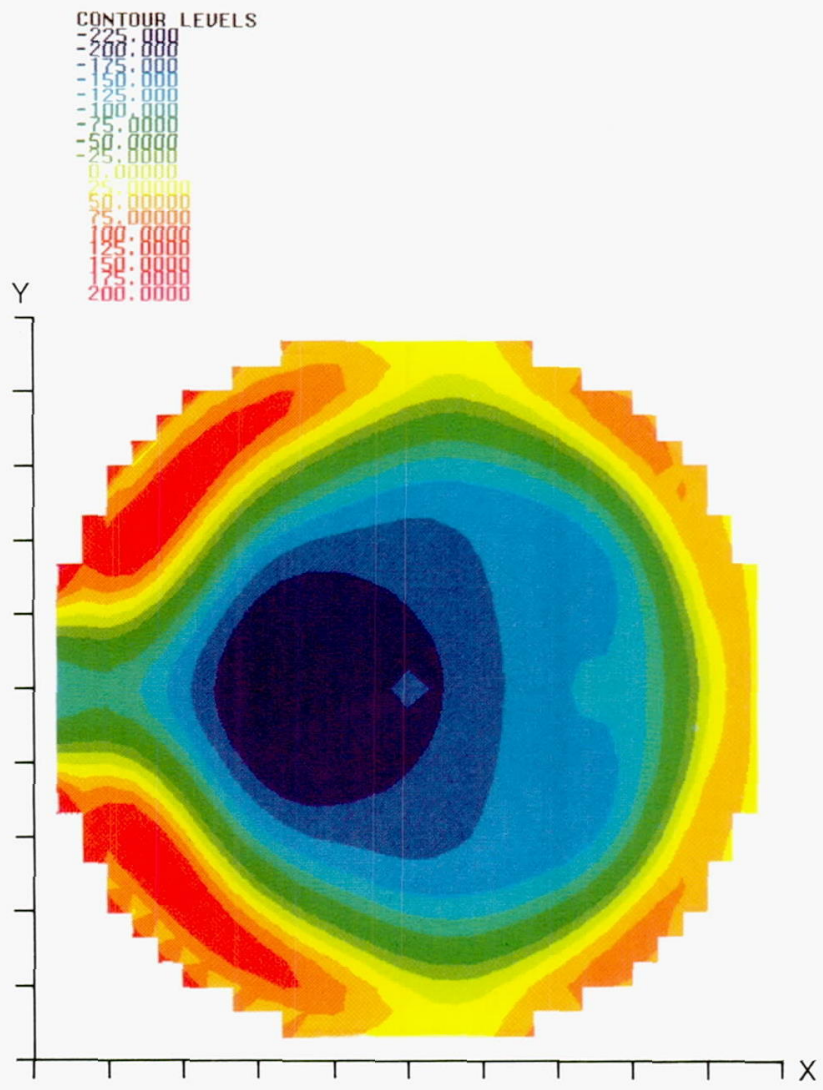

Figure 15. $-x y$-view is the same as in figure 12 except that each of the 29 projections used in the reconstruction was shifted one sample point in a random direction to illustrate the effects of the misregistration, one form of experimental error.

As mentioned earlier, reconstruction is considerably sensitive to misregistration of the projections. That is, it is important that the centers of each of the viewing direction sample planes should be projections of the same point, the origin. To illustrate the effects of poor registration, the same hologram used above was resampled after the data were deliberately misregistered. Each view was shifted by one sample point in a randomly chosen direction. In figures 15 to 17 the result is displayed. Although the general shape of the flask can be discerned in figure 15 , which shows the central $x y$-slice through the object, closer scrutiny reveals that the contour corresponding to the flask boundary is not well formed, showing elongation near the sides of the flask. The $x z$-plane, shown in figure 16 , is considerably more artifacted than figure 13, which shows the corresponding view for the properly registered data. This is especially true in the central region. The degradation is severe enough to mandate that serious attention be paid to the proper alignment of the views. Figure 17, the $x z$-central slice through the reconstruction from misregistered data, provides another illustration of the artifacting. 


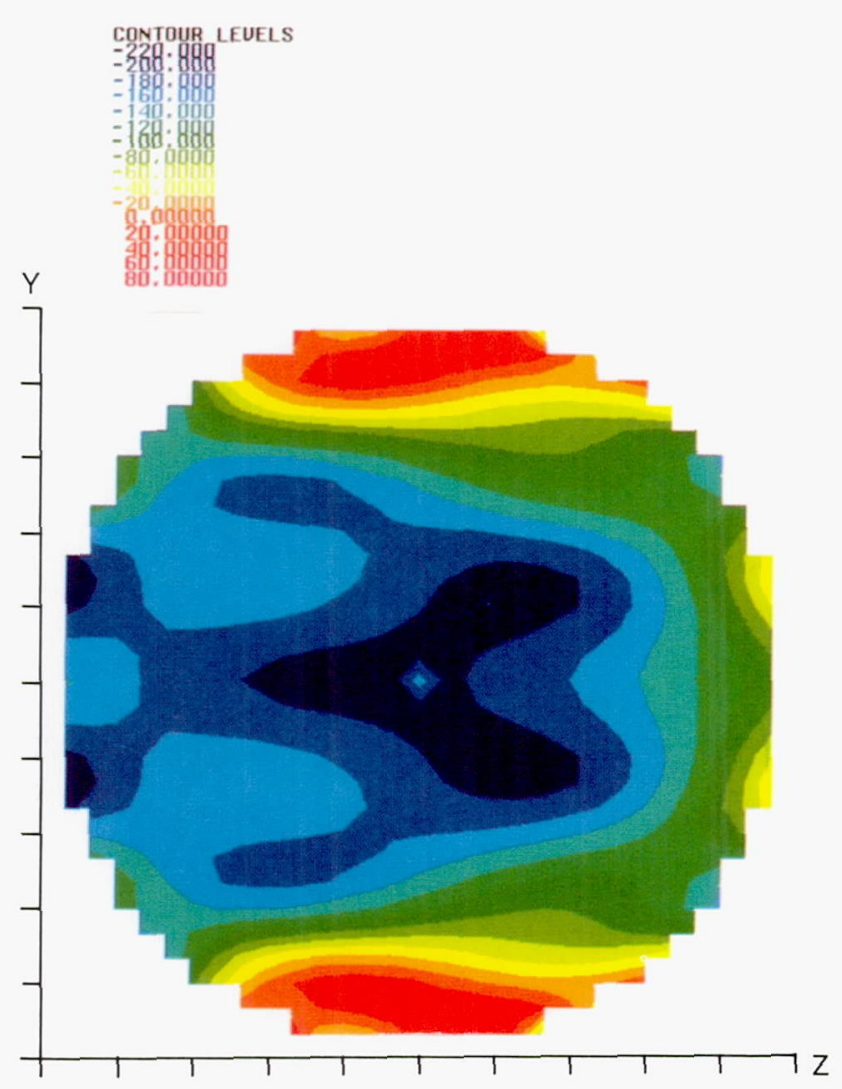

Figure 16.-This $y z$-slice is the same as in figure 13 except that the misregistered data set was used as input to the reconstruction algorithm.

\section{Conclusions}

Numerical and laboratory experiments were performed to test whether optical experimental techniques supplemented by three-dimensional tomographic data analysis could be used to measure scalar three-dimensional distributions. The numerical experiments predict, and the laboratory experiments confirm, that such reconstructions are extremely ill-posed. One $10^{\circ}$ viewing cone is clearly insufficient to accurately recover the object function. Additional data, such as from another cone of views, alternate laboratory techniques, or the application of constraints will be necessary before these methods can be used practically in a three-dimensional setting.

\section{Acknowledgment}

The authors wish to thank Ken Weiland of the NASA Lewis Research Center for his invaluable expertise and assistance in creating the holograms and for his incredible patience in scanning them.

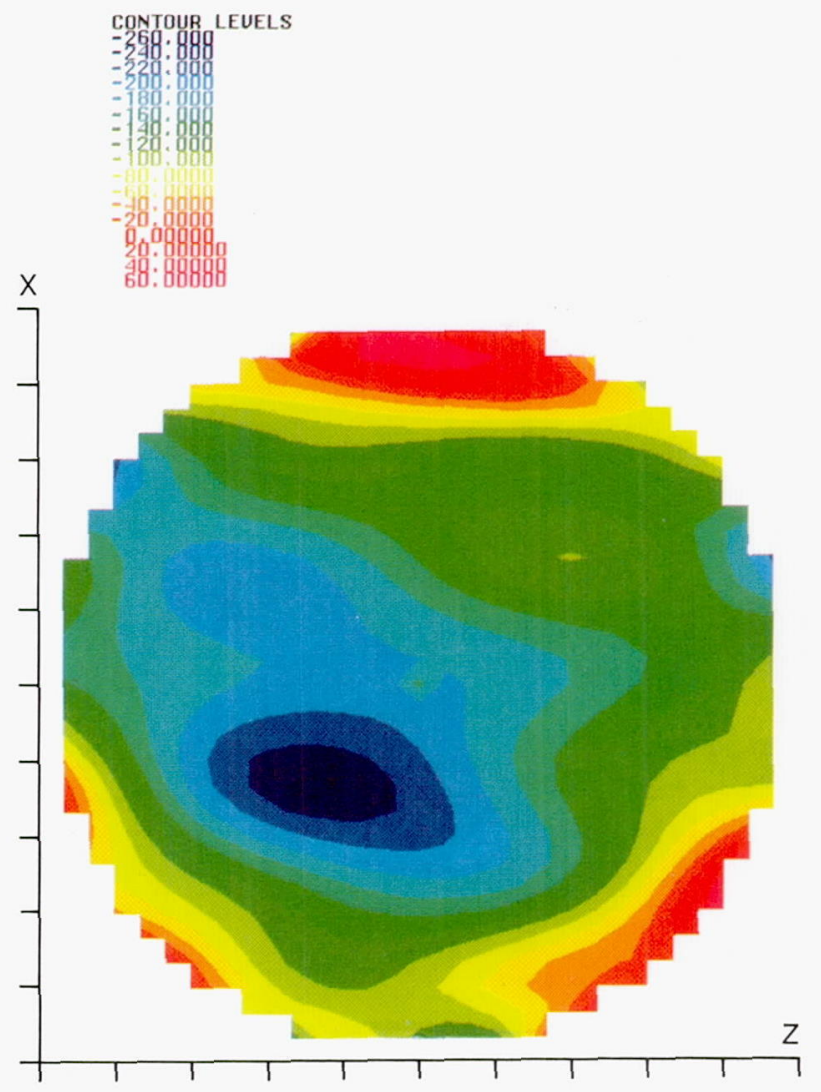

Figure 17.-This $x z$-slice is the same as in figure 14 except that the misregistered data set was used as input to the reconstruction algorithm.

\section{References}

1. Snyder, R; and Hesselink, L.: Optical Tomography for Flow Visualization of the Density Field Around a Revolving Helicopter Rotor Blade. Appl. Opt., vol. 23, 1984, pp. 3650-3656.

2. Strazisar, A.J.: Laser Fringe Anemometry for Aero Engine Components. Advanced Instrumentation for Aero Engine Components. AGARDCP-399, May 1986, pp. 6-1 to 6-32.

3. Eckbreth, A.C; Dobbs, G.M.; Stufflebeam, J.H.; and Tellex, P.A.: CARS Temperature and Species Measurements in Augmented Jet Engine Exhausts. Appl. Opt., vol. 23, 1984, pp. 1328-1339.

4. Hiller, B; and Hanson, R.K.; Simultaneous Planar Measurements of Velocity and Pressure Fields in Gas Flows Using Laser Induced Fluorescence. Appl. Opt., vol. 27, 1988, pp. 33-48.

5. Bryanston-Cross, P.J.: The Application of Holography as a Transonic Flow Diagnostic to Rotating Components in Turbomachinery. Advanced Instrumentation for Aero Engine Components. AGARD-CP-399, May 1986, pp. 32-1 to 32-22.

6. Stricker, J.; Keren, E.; and Kafri, O.: Axisymmetric Density Field Measurements by Moire Deflectometry. AIAA J., vol. 21, 1983, pp. $1767-1769$.

7. Howes, W.L.: Rainbow Schlieren and its Applications. Appl. Opt., vol. 23, 1984, pp. 2449-2460.

8. Gaydon, A.G.: The Spectroscopy of Flames. 2nd Edit., Wiley, New York, 1974 
9. Decker, A.J.: Holographic Interferometry with an Injection Seeded Nd:YAG Laser and Two Reference Beams. Appl. Opt., vol. 29, 1990, pp. 2696-2700.

10. Batchelor, G.K.: An Introduction to Fluid Dynamics. Cambridge, New York, 1967, pp. 164-167.

11. Deans, S.R.: The Radon Transform and Some of Its Applications. Wiley, New York, 1983, pp. 128-131.

12. Izen, S.H.: A Series Inversion for the X-Ray Transform in $n$ Dimensions. Inverse Prob., vol. 4, 1988, pp. 725-748.

13. Izen, S.H.: Inversion of the k-plane Transform by Orthogonal Function Series Expansions. Inverse Prob., vol. 5, 1989, pp. 181-202.

14. Izen, S.H.: Inversion of the X-Ray Transform from Data in a Limited Angular Range. Signal Processing, Part II: Control Theory and Applications. F.A. Grünbaum, J.W. Helton, and P. Khargonekar, eds., Springer Verlag, New York, 1990, pp. 275-284
15. Izen, S.H.: An Application for a Limited Solid Angle X-Ray Transform. To be published.

16. Louis, A.K.: Orthogonal Function Series Expansion and the Null Space of the Radon Transform. SIAM J. Math. Anal., vol. 15, 1984, pp. 621-633.

17. Natterer, F.: The Mathematics of Computed Tomography. Wiley, New York, 1986.

18. Riddick, J.A.; and Bunger, W.B.: Organic Solvents-Physical Properties and Methods of Purification. 3d Edit, Wiley, New York, 1970, p. 466.

19. Perry, R.H.; and Chilton, C.H.: Chemical Engineers' Handbook. 5th Edit., McGraw-Hill, New York, 1973, pp. 3-1 to 3-250.

20. Dandliker, R.: Heterodyne Holographic Interferometry. Progress in Optics. E. Wolf, ed., vol. 17, North-Holland, New York, 1980, pp. $1-84$ 


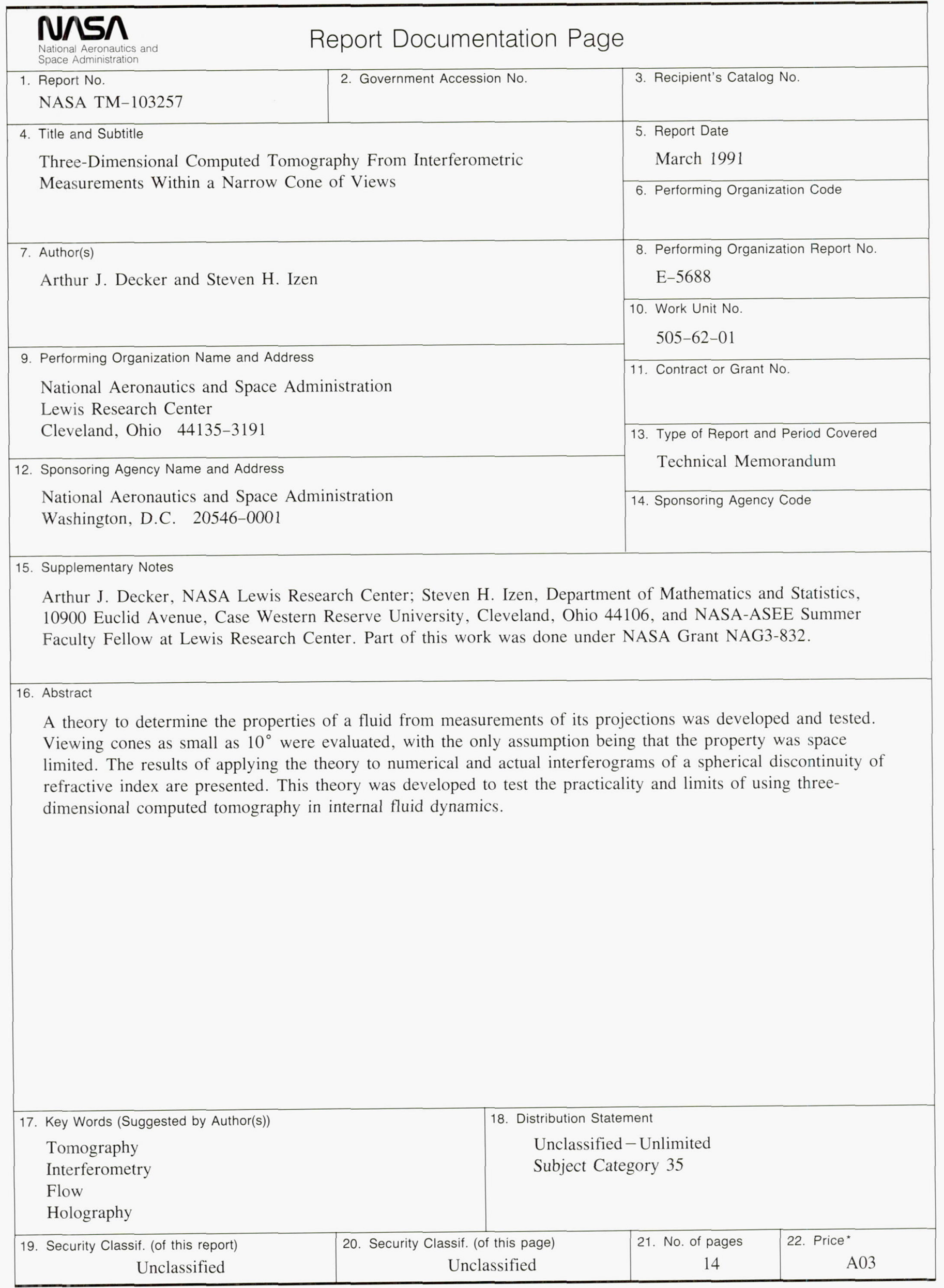

